\title{
Abundances of Planetary Nebulae BD+30 3639 and NGC 6543`
}

\author{
J. Bernard-Salas ${ }^{1,2}$, S. R. Pottasch ${ }^{2}$, P. R. Wesselius ${ }^{1}$, and W. A. Feibelman ${ }^{3}$ \\ 1 SRON National Institute for Space Research, PO Box 800, 9700 AV Groningen, The Netherlands \\ 2 Kapteyn Astronomical Institute, PO Box 800, 9700 AV Groningen, The Netherlands \\ ${ }^{3}$ Laboratory for Astronomy and Solar Physics, NASA-Goddard Space Flight Center, Greenbelt, MA 20771, USA \\ Received 28 February 2003 / Accepted 15 May 2003
}

\begin{abstract}
Infrared spectra taken with the Short Wavelength Spectrometer on board ISO and UV observations with IUE of planetary nebulae BD+30 3639 and NGC 6543 are presented. The extinction derived using infrared lines for BD+30 3639 is $E_{B-V}=0.34$, slightly higher than previous determinations. For NGC 6543 the extinction found from the hydrogen lines is $E_{B-V}=0.07$. Infrared, optical and ultraviolet data have been used to derive the physical parameters of the nebula. BD+30 3639 has an average $T_{\mathrm{e}}=8500 \mathrm{~K}$ and $N_{\mathrm{e}}=11000 \mathrm{~cm}^{-3}$. In the case of NGC $6543 \mathrm{a} T_{\mathrm{e}}=8200 \mathrm{~K}$ and $N_{\mathrm{e}}=5000 \mathrm{~cm}^{-3}$ have been found, in agreement with previous determinations. The element abundances have been derived and compared to those found in the Sun and O, B stars. This comparison gives a hint of the mass of the progenitor stars from which they evolved.
\end{abstract}

Key words. ISM: abundances - planetary nebulae: individual: BD+303639, NGC 6543 - infrared: ISM - ISM: lines and bands

\section{Introduction}

Both BD+303639 and NGC 6543 have a low temperature exciting star which translates into a spectrum dominated by lines of ions with low ionization potential. Past abundance studies of these two Planetary Nebulae (PNe) have been mostly focused on the optical and ultraviolet spectra. Nevertheless, some lines from important stages of ionization (those which contribute to the total abundance) of some elements lie in the infrared region of the spectrum and had to be inferred in previous studies. The inclusion of the infrared data from the ISO satellite allows us to accurately derive abundances of neon, argon, sulfur and chlorine. They also provide complementary information of other important stages of ionization such as $\mathrm{N}^{++}$and $\mathrm{O}^{3+}$ and have the advantage that they are not temperature dependent. The extinction plays a minor role in these lines. Other advantages of the infrared data have been briefly discussed by Beintema \& Pottasch (1999) and Bernard Salas et al. (2001). All these advantages have allowed us to derive accurate abundances for both PNe.

\section{1. $B D+303639$}

BD+303639 is a compact planetary nebula with a low extinction and a carbon Wolf-Rayet central star. It has been the object of many previous studies. Earlier observations with

Send offprint requests to: J. Bernard-Salas,

e-mail: J .Bernard@sron.rug.nl

^ Based on observations with ISO, an ESA project with instruments funded by ESA Member States (especially the PI countries: France, Germany, The Netherlands and the United Kingdom) and with the participation of ISAS and NASA. the International Ultraviolet Explorer (IUE) were reported by Torres-Peimbert \& Peña (1981). Pwa et al. (1986) derived ionic abundances for several ions, and using ionization corrections factors $(I C F)$, derived the ionic abundances for some elements. They found the dust-to-gas ratio to be $1.8 \%$, and determined an extinction for the interstellar component of $E_{B-V}=0.12$. Using the P Cygni profile of the C III $2297 \AA$ line they find a terminal velocity of $790 \pm 50 \mathrm{~km} \mathrm{~s}^{-1}$. A recent X-ray study of this nebula by Kastner et al. (2000) using the ACIS instrument on board the Chandra X-ray Observatory shows that the X-ray emission originates within the ionized gas, and is concentrated to one side. They found $T_{\mathrm{X}}=3 \times 10^{6} \mathrm{~K}$. The spectroscopic study reveals that the $\mathrm{X}$-ray emitting region may be enriched as a result of nuclear burning.

An optical and near-infrared study was made by Rudy et al. (1991), while Aller \& Hyung (1995) used high resolution observations from the Hamilton echelle spectrograph at Lick Observatory to study the physical conditions and abundances of the nebula. They found an electron density $\left(N_{\mathrm{e}}\right)$ of $10000 \mathrm{~cm}^{-3}$ and an electron temperature $\left(T_{\mathrm{e}}\right)$ of $8800 \mathrm{~K}$, and abundances very similar to solar. In spite of all these studies $\mathrm{BD}+303639$ still needs more attention. The SWS infrared spectrum shows silicates, which are related to oxygen rich $\mathrm{PNe}$, and Polycyclic Aromatic Hydrocarbon (PAHs) features, related to a carbonrich nebula. This remains a puzzle.

\subsection{NGC 6543}

PN NGC 6543 shows a very interesting morphology with bubbles and an extended halo. It has a central star classified as Of/WR. The diameter of the nebula is 19.5" (Hyung et al. 2000), and it has a halo with a radius of $165^{\prime \prime}$ 
(Middlemass et al. 1989). Castor et al. (1981) using IUE observations estimated the terminal velocity of the wind to be $2150 \mathrm{~km} \mathrm{~s}^{-1}$ and deduced a mass-loss rate of $1.0 \times 10^{-7} M_{\odot} / \mathrm{yr}$. Aller \& Czyzak (1979) studied a sample of $40 \mathrm{PNe}$, with emphasis on the weak lines, including NGC 6543. X-ray observations have been done by Guerrero et al. (2001) and they have detected a point source at the position of the central star of NGC 6543. They derived a X-ray temperature of a few times $10^{6} \mathrm{~K}$. A recent study, in terms of physical conditions and abundances, has been carried out by Hyung et al. (2000). They combine Hamilton echelle observations in two positions (east and north of the central star), with IUE data. They found an $T_{\mathrm{e}}=8000 \sim 8300 \mathrm{~K}$ and an $N_{\mathrm{e}}=5000 \mathrm{~cm}^{-3}$. They predicted a progenitor mass star of $\sim 1 M_{\odot}$. Manchado \& Pottasch (1989) presented observations of the central region and the halo of NGC 6543. For the central region they derived $N_{\mathrm{e}}=4000 \mathrm{~cm}^{-3}$ and $T_{\mathrm{e}}=7000 \mathrm{~K}$. For the halo they obtained a higher temperature and concluded that the radiation from the halo is thermal and not due to dust reflection. A detailed study of different parts of the halo can be found in Middlemass et al. (1989) comparing the abundances of the halo with the central core of the nebula.

\subsection{Plan of the paper}

In the next section the observations and reduction analysis is briefly discussed. Section 3 discusses the possibility for the need of aperture corrections. In Sect. 4 the infrared data is presented and the extinction derived. The optical and IUE data used in this study is listed in Sect. 5. The physical parameters are derived in Sect. 6 and the abundances in Sect. 7. Conclusions are given in the last section.

\section{Observations and data reduction}

\subsection{ISO observations}

\subsubsection{SWS observations}

The Short Wavelength Spectrometer on board ISO (de Graauw et al. 1996) covers the spectral range from 2.38 to $45.2 \mu \mathrm{m}$. ISO-SWS uses different size apertures for four different spectral regions. These apertures are $14^{\prime \prime} \times 20^{\prime \prime}, 14^{\prime \prime} \times 27^{\prime \prime}, 20^{\prime \prime} \times$ $27^{\prime \prime}$ and $20^{\prime \prime} \times 33^{\prime \prime}$ and cover respectively the wavelength intervals: $2.38-12.0 \mu \mathrm{m}, 12.0-27.5 \mu \mathrm{m}, 27.5-29 \mu \mathrm{m}$ and $29.0-45.2$ $\mu \mathrm{m}$.

The observations used for $\mathrm{BD}+303639$ and NGC 6543 correspond to the SWS01 observing template. The observation number for $\mathrm{BD}+303639$ is TDT $=86500540$. It is a slow observation, Speed 3, which corresponds to an exposure time of $3453 \mathrm{~s}$. It was centered at RA(2000) $19^{\mathrm{h}} 34^{\mathrm{m}} 45.2^{\mathrm{s}}$ and $\operatorname{Dec}(2000)+30^{\circ} 30^{\prime} 58.8^{\prime \prime}$. For NGC 6543 the observation used is TDT $=02400714$. This is a Speed 4 (slow) observation of 6544 s. It was centered at $\mathrm{RA}(2000) 17^{\mathrm{h}} 58^{\mathrm{m}} 33.4^{\mathrm{s}}$ and $\operatorname{Dec}(2000)+66^{\circ} 37^{\prime} 59.5^{\prime \prime}$. The observations were pointed at the center of the nebulae. The pointing errors for ISO are $\sim 1.5^{\prime \prime}$.

A description of the tools used to reduce the data can be found in the interactive analysis software package which is distributed by the SWS consortium, IA3 (de Graauw et al. 1996). Each wavelength range is covered by both an $u p$ and a down

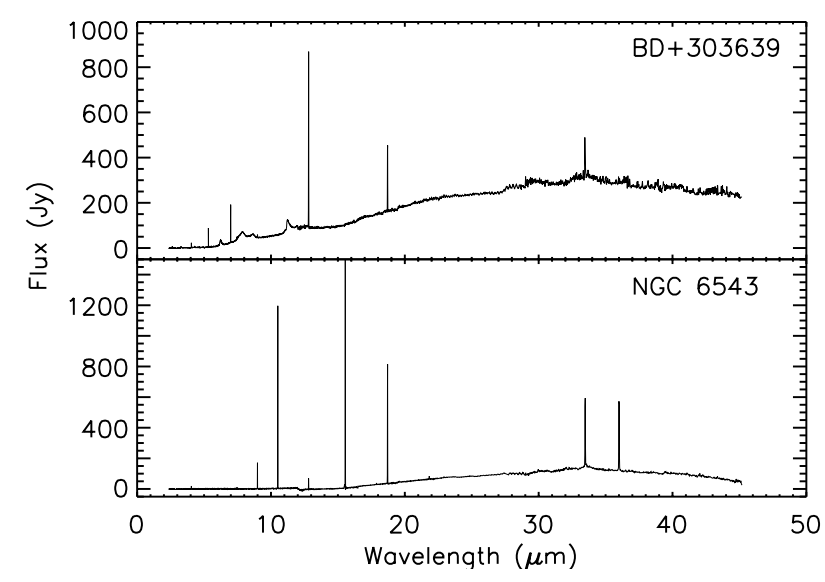

Fig. 1. SWS spectra of BD+303639 and NGC 6543.

scan. Each is sampled by twelve detectors. Both scans were reduced separately and combined to produce the spectra seen in Fig. 1. The reduction consists of several steps:

- Dark current subtraction. Sometimes some detectors were affected by memory effects and were not used when deriving the dark current.

- De-fringing: fringes were present in the $\mathrm{BD}+303639$ spectrum. The patterns are severe in bands $3 \mathrm{~A}, 3 \mathrm{C}, 3 \mathrm{D}$ and $3 \mathrm{E}$. These were removed using the IA tool fringes.

- Removal of glitches and bad data. The former are normally easily detected when comparing both scans because they are taken at different times. Band 4 is heavily affected and that makes it hard to tag the glitches.

- Flatfielding. The spectra of all detectors in each grating block were flatfielded to give the same signal.

- Sigma-clipping and rebinning. Data exceeding a $3 \sigma$ error were excluded; thereafter rebinned to a fixed resolution of 1000 .

Band $3 E$ (from 27.5 to $29 \mu \mathrm{m}$ ) for $\mathrm{BD}+303639$ and band 4 (from 29 to $45.2 \mu \mathrm{m}$ ) for NGC 6543 presented an offset with respect to the overall spectrum. This was due to the dark current which has an additive effect in the spectra. They were corrected applying a shift of $35 \mathrm{Jy}$ in $\mathrm{BD}+303639$ and $55 \mathrm{Jy}$ in NGC 6543. Apart from that the reduction did not present unusual problems.

\subsubsection{LWS observations}

Lines from the Long Wavelength Spectrometer (Clegg et al. 1996) spectra were also used. This instrument covers the wavelength range from 43 to $196 \mu \mathrm{m}$ and has a resolution that varies (depending on wavelength) from 140 to 330 . The aperture of the LWS is $80^{\prime \prime}$. The observational numbers are $\mathrm{TDT}=35501412$ for $\mathrm{BD}+303639$ and TDT $=25500701$ for NGC 6543 and correspond to the AOT01 observing template. The lines used were those at 51.8 and $88.3 \mu \mathrm{m}$ (O III) and at $57.3 \mu \mathrm{m}(\mathrm{N}$ III) and were taken from Bernard-Salas \& Tielens (2003, in prep.). 


\subsection{Ultraviolet and optical observations}

The ultraviolet spectra used in this paper were taken with the International Ultraviolet Explorer (IUE). The aperture of the IUE is an ellipse of $10^{\prime \prime} \times 23^{\prime \prime}$.

In $\mathrm{BD}+303639$ the [WR] central star contaminates the nebular ultraviolet spectrum; therefore only the uncontaminated nebular C II of Torres-Peimbert \& Peña (1981), was used. The C III line at $190.9 \mathrm{~nm}$ is produced both by the star and the nebula. Torres-Peimbert \& Peña (1981) assume that only half of the intensity is of nebular origin when deriving the ionic abundance, but this is uncertain. The nebular contribution can be separated from the stellar contribution using high resolution spectra. There are only two of these IUE observations in which this line is not saturated. Due to the quality of the spectra the SWP51870 observation centered at $\operatorname{RA}(2000) 19^{\mathrm{h}} 34^{\mathrm{m}} 45.2^{\mathrm{s}}$ and $\operatorname{Dec}(2000)+30^{\circ} 30^{\prime} 59.7^{\prime \prime}$ with a exposure time of $10200 \mathrm{~s}$. was used to give the intensity for this line. This appears as a narrow component above the broad stellar line and is tabulated in Table 5. The optical data was taken with a slot size of $1.2^{\prime \prime} \times 4.0^{\prime \prime}$ (Aller \& Hyung 1995). This slot does not contain the whole nebula, and therefore each line flux has been scaled to the total flux using the entire $\mathrm{H}_{\beta}$ flux $\left(93.3 \times 10^{-12} \mathrm{erg} \mathrm{cm}^{-2} \mathrm{~s}^{-1}\right)$, and we have assumed here that they are representative of the whole nebula.

For NGC 6543 high and low resolution spectra were used, labeled LWP24732 and SWP54878 for the low resolution spectra and SWP03323 and LWP07710 for the high resolution. All were centered on the central star $\left(\operatorname{RA}(2000) 17^{\mathrm{h}} 58^{\mathrm{m}} 33.4^{\mathrm{s}}\right.$ and $\left.\operatorname{Dec}(2000)+66^{\circ} 37^{\prime} 58.8^{\prime \prime}\right)$ and have exposures times of $10200,120,120,18000$, and 12600 s. respectively. The optical data were taken from several authors: Manchado \& Pottasch (1989), Middlemass et al. (1989), Aller \& Czyzak (1979) and Hyung et al. (2000). The latter reference observed BD+30 3639 in two different regions of the nebula, north and east of the central star. Since the entire nebula is not measured the flux has again been scaled using the $\mathrm{H}_{\beta}$ flux, in this case $245.5 \times$ $10^{-12} \mathrm{erg} \mathrm{cm}^{-2} \mathrm{~s}^{-1}$ (Acker et al. 1992).

\section{Aperture corrections}

$\mathrm{BD}+303639$ is a compact object. Mid-infrared spectral images have been taken with ISOCAM by Persi et al. (1999), and show an effective size of $6.2^{\prime \prime} \times 5.7^{\prime \prime}$ in the [Ne II] image. This size is much smaller than any of the ISO-SWS apertures, therefore the whole flux is seen in all apertures. The same applies to the IUE aperture, which is an ellipse of $10^{\prime \prime} \times 23^{\prime \prime}$ (similar to the smallest SWS aperture).

The case of NGC 6543 is more troublesome. Most of the emission comes from a region of about $19.5^{\prime \prime}$ in diameter. This is slightly bigger than the smallest ISO-SWS and IUE apertures. It is therefore likely that these observations do not see the whole nebula and aperture corrections are needed. This correction can be derived by means of the observed hydrogen lines in the small SWS aperture. The extinction corrected fluxes $\left(E_{B-V}=0.07\right.$, see Sect. 4.2) can be used together with the theoretical predictions by Hummer \& Storey (1987) to predict the extinction corrected $\mathrm{H}_{\beta}$ flux. The average of these predictions
Table 1. Comparison of IRAS and ISO-SWS fluxes, in units of $10^{-12} \mathrm{erg} \mathrm{cm}^{-2} \mathrm{~s}^{-1}$ for NGC 6543 .

\begin{tabular}{clcccc}
\hline \hline $\begin{array}{c}\lambda \\
(\mu \mathrm{m})\end{array}$ & Ion & \multicolumn{2}{c}{ SWS } & IRAS & IRAS/SWS \\
\cline { 3 - 4 } & & Aperture & Flux & Flux & \\
\hline 8.994 & Ar III & $14^{\prime \prime} \times 20^{\prime \prime}$ & 68.8 & 90 & 1.31 \\
10.52 & S IV & $14^{\prime \prime} \times 20^{\prime \prime}$ & 385.2 & 430 & 1.11 \\
12.81 & Ne II & $14^{\prime \prime} \times 27^{\prime \prime}$ & 22.2 & 20 & 0.90 \\
15.56 & Ne III & $14^{\prime \prime} \times 27^{\prime \prime}$ & 631.8 & 530 & 0.84 \\
18.70 & S III & $14^{\prime \prime} \times 27^{\prime \prime}$ & 132.0 & 180 & 1.36 \\
\hline
\end{tabular}

is $236 \times 10^{-12} \mathrm{erg} \mathrm{cm}^{-2} \mathrm{~s}^{-1}$ while the observed $\mathrm{H} \beta$ (corrected for extinction) is $310 \times 10^{-12} \mathrm{erg} \mathrm{cm}^{-2} \mathrm{~s}^{-1}$. This indicates that some flux, a factor 1.3 , is missing in the smallest aperture. This factor has been used to correct all the SWS lines measured in the smallest aperture.

IRAS-LRS measurements (Pottasch et al. 1986) with a diaphragm of $6^{\prime} \times 5^{\prime}$ can also be used to indicate whether a correction factor (Table 1) in the small and larger SWS apertures is needed. Although the fluxes in Table 1 are an average of over 200 observations (reducing therefore the uncertainty) the LRS lines fluxes are normally good to $\sim 30$ to $50 \%$ and that is why this comparison can be used only as an indication. For Ar III and S IV it seems that some flux is missing in the smallest SWS aperture. The factors 1.1 and 1.3 agree more or less with the factor derived using the hyrogen lines. From the neon lines it seems that in the larger apertures apparently the whole flux is seen. The IRAS fluxes for the S IV is uncertain because the poor spectral resolution makes it appear as a broad line in which it is difficult to define the continuum. In any case this line agrees within errors with the SWS. Persi et al. (1999) measured a handful of lines in the region between 5 and $16.5 \mu \mathrm{m}$. Although these measurements have a poor spectral resolution the agreement of the Ar III and Ne II fluxes with those of IRAS is quite good, but the fluxes of the S IV and Ne III differ by large amount.

In the case of the IUE that has a similar aperture as the SWS smallest aperture and was centered at the same position, an aperture correction factor of 1.3 has also been assumed. The reader should bear in mind that this correction is probably good to \pm 0.1 and not the same for all species. The goodness of this assumption is important since it affects the abundance determination of the ions whose abundance is derived using ultraviolet or infrared $(<12 \mu \mathrm{m})$ lines.

\section{Infrared spectrum and extinction}

Line fluxes were determined using the line fitting routine in the ISO Spectroscopy Analysis Package (ISAP).

\section{1. $B D+303639$}

Eleven lines were measured with SWS, listed in Table 2. For some unseen lines it is useful to deduce an upper limit to the flux. Upper limits for the lines [S IV] $10.51 \mu \mathrm{m}$, [Cl IV] $11.76 \mu \mathrm{m}$ and [N III] $57.3 \mu \mathrm{m}$ were derived using a model of a synthetic Gaussian, with the width corresponding to the 
Table 2. Infrared line intensities for BD+30 3639. All fluxes are in units of $10^{-12} \mathrm{erg} \mathrm{cm}^{-2} \mathrm{~s}^{-1}$. In the last column the unreddened flux is given using $E_{B-V}=0.34$.

\begin{tabular}{clcc}
\hline \hline$\lambda(\mu \mathrm{m})$ & Ident. & Flux $^{\text {obs }}$ & Flux $^{\text {corr }}$ \\
\hline 2.625 & H I Br $\beta$ & 13.30 & 14.01 \\
2.872 & H I 5-11 & $1.30:$ & $1.36:$ \\
3.039 & H I 5-10 & 1.71 & 1.78 \\
3.740 & H I Pf $\gamma$ & 3.33 & 3.42 \\
4.051 & H I Br $\alpha$ & 25.1 & 25.7 \\
6.984 & [Ar II] & 139.0 & 140.5 \\
7.458 & H I Pf $\alpha$ & 7.69 & 7.77 \\
8.991 & {$[$ Ar III] } & 6.10 & 6.40 \\
10.51 & {$[\mathrm{~S} \mathrm{IV}]$} & $<1.77$ & $<1.86$ \\
11.76 & {$[\mathrm{Cl}$ IV] } & $<1.67$ & $<1.72$ \\
12.81 & {$[\mathrm{Ne}$ II $]$} & 298.9 & 304.9 \\
15.55 & {$[\mathrm{Ne}$ III] } & $<2.42$ & $<2.46$ \\
18.70 & {$[\mathrm{~S} \mathrm{III]}$} & 60.4 & 61.7 \\
33.48 & {$[\mathrm{~S} \mathrm{III}]$} & 12.18 & 12.28 \\
$57.30^{\dagger}$ & {$[\mathrm{N} \mathrm{III]}$} & $<3.20$ & $<3.21$ \\
$122.0^{\dagger}$ & {$[\mathrm{N} \mathrm{II}]$} & $1.08:$ & $1.08:$ \\
\hline
\end{tabular}

$\dagger$ From the LWS spectrum.

: Noisy.

Table 3. Extinction for $\mathrm{BD}+303639$. The predicted $\mathrm{H}_{\beta}$ fluxes have been derived using the theoretical fluxes from Hummer \& Storey (1987) (see text for explanation).

\begin{tabular}{cccccc}
\hline \hline Ident. & Transition & $\begin{array}{c}\lambda \\
(\mu \mathrm{m})\end{array}$ & Flux $^{\sharp}$ & $\begin{array}{c}\text { Predict. } \\
\mathrm{H}_{\beta}\end{array}$ & $C_{\mathrm{H}_{\beta}}$ \\
\hline $\mathrm{Br} \alpha$ & $5 \rightarrow 4$ & 4.052 & 25.1 & 304 & 0.51 \\
$\mathrm{Br} \beta$ & $6 \rightarrow 4$ & 2.626 & 13.3 & 283 & 0.48 \\
$\operatorname{Pf} \alpha$ & $6 \rightarrow 5$ & 7.459 & 7.69 & 292 & 0.50 \\
$\mathrm{Pf} \gamma$ & $8 \rightarrow 5$ & 3.740 & 3.33 & 303 & 0.51 \\
$\mathrm{HI}$ & $13 \rightarrow 5$ & 3.039 & 1.71 & 311 & 0.52 \\
\hline
\end{tabular}

resolution, and a height of three times the RMS (deviation from the average). The upper limit to the flux for the $[\mathrm{N} \mathrm{III]}$ ion at $57.3 \mu \mathrm{m}$ was computed from LWS data.

The extinction for BD+30 3639 has been derived by comparing the observed $\mathrm{H}_{\beta}$ flux with the one predicted by extrapolating the hydrogen lines in the infrared spectrum (see Table 3 ). The hydrogen line at $2.872 \mu \mathrm{m}$ has been avoided in this calculation because it is noisy.

The predicted $\mathrm{H}_{\beta}$ fluxes from the infrared lines (Col.5) have been derived using the theoretical fluxes given by Hummer \& Storey (1987) for a black body case B. The comparison was made at a temperature of $8500 \mathrm{~K}$ and an electron density of $11000 \mathrm{~cm}^{-3}$, physical parameters expected for BD+303639 (see Sects. 6.1 and 6.2). The predicted $\mathrm{H}_{\beta}$ fluxes have been compared to the observed one of $93.3 \times 10^{-12} \mathrm{erg} \mathrm{cm}^{-2} \mathrm{~s}^{-1}$ by Acker et al. (1992). The extinction $C_{\mathrm{H}_{\beta}}\left(\right.$ where $C_{\mathrm{H}_{\beta}}=\log \left(\frac{F\left(\mathrm{H}_{\beta}\right)_{\text {predicted }}}{\left.F\left(\mathrm{H}_{\beta}\right)_{\text {observed }}\right)}\right)$ is shown in the last column of Table 3 . The values are very similar and average to $C_{\mathrm{H}_{\beta}}=0.50$, that leads to $E_{B-V}=0.34$. All the lines (infrared, optical and ultraviolet) have been corrected for extinction using this value and the extinction law of Fluks et al. (1994).
Table 4. Infrared measured line intensities for NGC 6543. In the last column the dereddened and aperture corrected fluxes (Flux ${ }^{\text {corr }}$ ) are given. The $E_{B-V}=0.07$, and the aperture correction factor is 1.3 for ions measured with the smallest ISO SWS aperture $(<12 \mu \mathrm{m})$.

\begin{tabular}{|c|c|c|c|}
\hline$\lambda(\mu \mathrm{m})$ & Ident. & Flux obs & Flux ${ }^{\text {corr }}$ \\
\hline 2.625 & H I $(\mathrm{Br} \beta)$ & 10.6 & 13.9 \\
\hline 3.038 & Н І (10-5) & 1.56 & 2.04 \\
\hline 3.296 & H I $(\operatorname{Pf} \delta)$ & 1.41: & 1.85 \\
\hline 3.739 & H I (Pf $\gamma)$ & 2.57 & 3.36 \\
\hline 4.051 & Н I $(\mathrm{Br} \alpha)$ & 17.7 & 23.2 \\
\hline 4.486 & Mg IV & $<1.88$ & $<2.45$ \\
\hline 4.653 & $\mathrm{HI}_{\mathrm{I}}(\operatorname{Pf} \beta)$ & 4.32 & 5.64 \\
\hline 6.984 & [Ar II] & 3.60: & 4.69 \\
\hline 7.312 & [Na III] & 3.28 & 4.27 \\
\hline 7.459 & Н І $(\operatorname{Pf} \alpha)$ & 5.79 & 7.54 \\
\hline 7.502 & H I (8-6) & 2.43 & 3.17 \\
\hline 8.990 & [Ar III] & 68.8 & 90.3 \\
\hline 10.51 & [S IV] & 385.2 & 505.6 \\
\hline 12.81 & {$[\mathrm{Ne}$ II $]$} & 22.2 & 22.3 \\
\hline 15.55 & [Ne III] & 631.8 & 634.0 \\
\hline 18.70 & [S III] & 132.0 & 132.6 \\
\hline 19.06 & H I (8-7) & 1.82 & 1.83 \\
\hline 21.83 & [Ar III] & 4.41 & 4.43 \\
\hline 22.32 & Н I, He II & 0.41: & 0.41 \\
\hline 25.89 & O IV & $<0.36$ & $<0.36$ \\
\hline 33.48 & [S III] & 51.2 & 51.3 \\
\hline 34.81 & [Si II $]$ & 1.21: & 1.21: \\
\hline 36.01 & [Ne III] & 52.8 & 52.9 \\
\hline $51.80^{\dagger}$ & {$[\mathrm{O} \mathrm{III}]$} & 504.7 & 505.2 \\
\hline $57.31^{\dagger}$ & {$[\mathrm{N}$ III] } & 120.6 & 126.7 \\
\hline $88.33^{\dagger}$ & [O III] & 144.3 & 144.3 \\
\hline
\end{tabular}

†From the LWS spectrum.

: Noisy.

Previous studies deduced a similar extinction for BD+30 3639 . Rudy et al. (1991) found an extinction of $C_{\mathrm{H}_{\beta}}=0.65$ and 0.39 from the optical the infrared data respectively. Aller \& Hyung (1995) derived $C_{\mathrm{H}_{\beta}}=0.40$, while Torres-Peimbert \& Peña (1981) found 0.44. It is worth noting that Hummer \& Storey (1987) give theoretical predictions for temperatures of 7500 and $10000 \mathrm{~K}$, and densities of $10^{4}$ and $10^{5} \mathrm{~cm}^{-3}$ and we have interpolated these.

\subsection{NGC 6543}

In Table 4 the twenty one lines measured with SWS are tabulated. Upper limits for Mg IV at $4.49 \mu \mathrm{m}$ and O IV at $25.9 \mu \mathrm{m}$ were also derived. Three LWS lines were also used. The strongest lines correspond to Ne III and S IV. Si II, although faint, is also clearly detected.

It is not possible to derive the extinction in the same way as for BD+30 3639 because the hydrogen lines have been measured with the smallest aperture, which as discussed in Sect. 2, may miss some nebular flux. The expected $\mathrm{H}_{\beta}$ can be found from the $6 \mathrm{~cm}$ radio emission using the following equation:

$F\left(\mathrm{H}_{\beta}\right)=\frac{S_{v}}{2.82 \times 10^{9} t^{0.53}\left(1+\frac{\mathrm{He}^{+}}{\mathrm{H}^{+}}+3.7 \frac{\mathrm{He}^{++}}{\mathrm{H}^{+}}\right)}$. 
Table 5. Ultraviolet (IUE) and optical line fluxes in units of $10^{-12} \mathrm{erg} \mathrm{cm}^{-2} \mathrm{~s}^{-1}$ for $\mathrm{BD}+303639$. The optical lines are from Aller \& Hyung (1995).

\begin{tabular}{|c|c|c|c|}
\hline$\lambda(\mathrm{nm})$ & Ident. & Flux $^{\text {obs }}$ & Flux $^{\text {corr }}$ \\
\hline $190.9^{\dagger}$ & C III] & 1.76 & 21.7 \\
\hline $232.6^{\star}$ & C II & 19.7 & 292.5 \\
\hline 372.6 & [O II $]$ & 98.8 & 417.3 \\
\hline 372.9 & {$[\mathrm{O} \mathrm{II}]$} & 40.2 & 169.6 \\
\hline 406.9 & [S II] & 6.04 & 23.6 \\
\hline 407.6 & [S II] & 1.62 & 6.31 \\
\hline 447.1 & He I & 0.77 & 2.73 \\
\hline $486.1^{\sharp}$ & $\mathrm{H}_{\beta}$ & 93.3 & 293.9 \\
\hline 493.3 & [O III] & 0.06 & 0.18 \\
\hline 495.9 & [O III] & 2.09 & 6.39 \\
\hline 519.9 & {$[\mathrm{~N} \mathrm{I}]$} & 0.24 & 0.68 \\
\hline 520.1 & {$[\mathrm{NI}]$} & 0.18 & 0.51 \\
\hline 551.8 & [Cl III] & 0.12 & 0.31 \\
\hline 553.8 & [Cl III $]$ & 0.37 & 0.97 \\
\hline 575.4 & {$[\mathrm{~N}$ II $]$} & 5.15 & 12.89 \\
\hline 587.6 & $\mathrm{He} \mathrm{I}$ & 4.06 & 9.94 \\
\hline 630.0 & [O I] & 1.88 & 4.30 \\
\hline 631.2 & [S III] & 1.32 & 3.01 \\
\hline 636.3 & [O I] & 0.71 & 1.61 \\
\hline 658.3 & {$[\mathrm{~N}$ II $]$} & 457.0 & 1002.9 \\
\hline 671.6 & [S II] & 5.18 & 11.12 \\
\hline 673.1 & [S II] & 11.17 & 24.0 \\
\hline 858.1 & {$[\mathrm{Cl} \mathrm{II}]$} & 1.01 & 1.68 \\
\hline 912.6 & [Cl II] & 0.27 & 0.43 \\
\hline
\end{tabular}

$\dagger$ From the IUE observations (see Sect. 2.2).

* Taken from Torres-Peimbert \& Peña (1981).

$\sharp$ From Acker et al. (1992).

In this equation the flux density $S_{v}=0.873 \mathrm{Jy}$ at $5 \mathrm{GHz}$ (George et al. 1974), $t$ is the electron temperature in units of $10^{4} \mathrm{~K}$ ( 0.82 for this nebula) and $2.82 \times 10^{9}$ is a conversion factor to give the $F\left(\mathrm{H}_{\beta}\right)$ in $\mathrm{erg} \mathrm{cm}^{-2} \mathrm{~s}^{-1}$. The helium abundances used in this calculation are those of Table 10 . The predicted $\mathrm{H}_{\beta}$ flux is then $307.8 \times 10^{-12} \mathrm{erg} \mathrm{cm}^{-2} \mathrm{~s}^{-1}$. The measured $\mathrm{H}_{\beta}$ flux by Acker et al. (1992) is $245.5 \times 10^{-12} \mathrm{erg} \mathrm{cm}^{-2} \mathrm{~s}^{-1}$. This leads to $C_{\mathrm{H}_{\beta}}=0.1$ or $E_{B-V}=0.07$ which we shall use in the remainder of this paper.

\section{The visual and the ultraviolet spectrum}

Optical and ultraviolet lines have been used to provide more information on ionization stages of some ions, especially carbon, oxygen and nitrogen.

\section{1. $B D+303639$}

The optical and ultraviolet data have been taken from Aller \& Hyung (1995) and Torres-Peimbert \& Peña (1981) respectively. In Table 5 the ions used are listed. The dereddened fluxes have been derived using an extinction of $E_{B-V}=0.34$, as derived from the infrared lines (see the previous section). Several lines of the same ion were sometimes used to confirm the results of the ionic abundances.
Table 6. Ultraviolet (IUE) line fluxes in units of $10^{-12} \mathrm{erg} \mathrm{cm}^{-2} \mathrm{~s}^{-1}$ for NGC 6543. In the last column the fluxes have been corrected for interstellar extinction using $E_{B-V}=0.07$ and an aperture correction factor of 1.3 has been used.

\begin{tabular}{|c|c|c|c|}
\hline$\overline{\lambda(\mathrm{nm})}$ & Ident. & Flux ${ }^{\text {obs }}$ & Flux $^{\text {corr }}$ \\
\hline \multicolumn{4}{|c|}{ High Resolution } \\
\hline $137.1^{s}$ & $\mathrm{OV}$ & 2.64 & 5.99 \\
\hline 148.3 & N IV] & 0.05 & 0.10 \\
\hline 148.7 & $\mathrm{~N}$ IV] & 0.03 & 0.07 \\
\hline $155.2^{s}$ & C IV & 60.87 & 133.67 \\
\hline 166.0 & O III] & 0.34 & 0.74 \\
\hline 166.6 & O III] & 0.98 & 2.13 \\
\hline 174.6 & $\mathrm{~N}$ III] & 0.22 & 0.47 \\
\hline 174.8 & N III] & 0.21 & 0.45 \\
\hline 174.9 & $\mathrm{~N}$ III] & 0.66 & 1.42 \\
\hline 190.6 & C III] & 8.34 & 18.19 \\
\hline 190.8 & C III] & 7.66 & 16.71 \\
\hline 229.7 & C III & 1.41 & 3.25 \\
\hline 232.1 & O III & 0.07 & 0.15 \\
\hline 232.5 & $\mathrm{C}$ II] & 0.71 & 1.61 \\
\hline 232.7 & $\mathrm{C}$ II $]$ & 1.19 & 2.70 \\
\hline 283.7 & O IV]? & 1.24 & 2.37 \\
\hline 302.5 & O III & 0.31 & 0.58 \\
\hline 304.7 & O III & 0.55 & 1.02 \\
\hline $313.3^{\natural}$ & O III & 0.46 & 0.85 \\
\hline $320.3^{s}$ & He II & 0.28 & 0.51 \\
\hline \multicolumn{4}{|c|}{ Low Resolution } \\
\hline $124.4^{s}$ & $\mathrm{NV}$ & 57.7 & 140.28 \\
\hline 131.0 & $?$ & 11.9 & 27.83 \\
\hline $131.7^{b}$ & $?$ & 7.30 & 17.01 \\
\hline $137.6^{s}$ & $\mathrm{OV}$ & 29.9 & 67.66 \\
\hline 140.9 & $?$ & 14.6 & 32.61 \\
\hline $155.2^{s}$ & C IV & 56.4 & 123.85 \\
\hline $164.1^{s}$ & He II & 17.4 & 37.96 \\
\hline 166.2 & O III] & 4.50 & 9.78 \\
\hline 172.1 & N IV] & 11.0 & 23.69 \\
\hline 174.5 & N III] & 1.90 & 4.08 \\
\hline 175.9 & $?$ & 2.50: & 5.36 \\
\hline 181.4 & [Ne III] & 3.40 & 7.29 \\
\hline 190.7 & C III] & 16.9 & 36.86 \\
\hline 229.6 & $\mathrm{C}$ III & 4.90 & 11.30 \\
\hline 231.1 & $\mathrm{C}$ II $]+\mathrm{O}$ III & 7.10 & 16.25 \\
\hline 232.9 & $\mathrm{C}$ II] & 3.70 & 8.38 \\
\hline 241.9 & [Ne IV] & 2.20 & 4.74 \\
\hline 251.0 & He II & 2.00 & 4.15 \\
\hline 267.7 & $?$ & 2.30 & 4.52 \\
\hline 279.0 & {$[\mathrm{Ar} \mathrm{v}]+[\mathrm{Mg} \mathrm{v}]$} & 2.10 & 4.04 \\
\hline 283.5 & $\mathrm{C}$ II] & 3.00 & 5.73 \\
\hline $292.2^{\dagger}$ & $\mathrm{Mg} \mathrm{V}$ & 0.70 & 1.32 \\
\hline $298.7^{b}$ & {$[\mathrm{Ne} \mathrm{V}]$ ? } & 2.20 & 4.12 \\
\hline 318.8 & Si II, He I & 2.30 & 4.20 \\
\hline 320.9 & He II & 1.50 & 2.73 \\
\hline
\end{tabular}

${ }^{s}$ Stellar line.

${ }^{\natural}$ Affected by reseau.

${ }^{b}$ Blended.

${ }^{\dagger}$ May be present.

: Noisy.

\subsection{NGC 6543}

The ultraviolet lines measured in the high and low resolution IUE spectra are given in Table 6. Some lines are contaminated by the central star (indicated in the table) and were obviously 
Table 7. Optical line fluxes for NGC 6543. In Col. 8 the average fluxes from MP, M, AC and H-east are given (see Sect. 4.2 for details). All the fluxes from Cols. 3 to 8 have been corrected for extinction using $C_{\mathrm{H}_{\beta}}=0.2$ (as are given in the literature) and are relative to $\mathrm{H}_{\beta}$. In this paper a lower extinction is found. The last column lists the average fluxes corrected for extinction using $C_{\mathrm{H}_{\beta}}=0.1\left(E_{B-V}=0.07\right)$ and in units of $10^{-12} \mathrm{erg} \mathrm{cm}^{-2} \mathrm{~s}^{-1}$.

\begin{tabular}{|c|c|c|c|c|c|c|c|c|}
\hline \multirow{2}{*}{$\begin{array}{c}\lambda \\
(\mathrm{nm})\end{array}$} & \multirow[t]{2}{*}{ Ident. } & \multicolumn{5}{|c|}{ Flux $^{\dagger}$} & \multirow[b]{2}{*}{ Average } & \multirow[t]{2}{*}{$\overline{\overline{\text { Flux }}}$} \\
\hline & & MP & Midd & $\mathrm{AC}$ & H-East & H-North & & \\
\hline 372.6 & {$[\mathrm{O}$ II $]$} & $15.5^{b}$ & $23.9^{b}$ & $21.0^{b}$ & 8.292 & 23.53 & 8.292 & 24.35 \\
\hline 372.9 & [O II $]$ & & & & 3.471 & 10.15 & 3.471 & 10.20 \\
\hline 386.9 & [Ne III] & 60.42 & & 51.0 & 48.35 & 38.67 & 53.26 & 157.4 \\
\hline 396.8 & [Ne III] & 35.65 & & 32.0 & 18.62 & 16.56 & 28.76 & 85.4 \\
\hline 406.9 & [S II] & & & & 0.540 & 2.108 & 0.54 & 1.61 \\
\hline 407.6 & [S II] & & & & 0.358 & 0.848 & 0.385 & 1.15 \\
\hline 436.3 & [O III] & 1.49 & 1.80 & 2.2 & 2.051 & 1.581 & 1.89 & 5.71 \\
\hline 447.2 & $\mathrm{He} \mathrm{I}$ & 6.20 & 5.30 & 6.02 & 6.018 & 5.158 & 5.88 & 17.9 \\
\hline 468.6 & He II & & & & 0.050 & & 0.05 & 0.15 \\
\hline 471.1 & [ArIV] & & & 1.3 & 0.935 & 0.284 & 1.12 & 3.45 \\
\hline 474.0 & [ArIV] & & & & 0.910 & 0.360 & 0.91 & 2.81 \\
\hline 486.1 & $\mathrm{HI}\left(\mathrm{H}_{\beta}\right)$ & 100 & 100 & 100 & 100 & 100 & 100 & 310.9 \\
\hline 495.9 & [O III] & 264.1 & 227.3 & & 218.8 & 172.2 & 236.7 & 740.4 \\
\hline 500.7 & [O III] & 774.7 & 663.0 & 675.0 & 704.3 & 518.5 & 704.2 & 2209 \\
\hline 519.2 & [Ar III] & & & & 0.055 & 0.051 & 0.055 & 0.17 \\
\hline 551.8 & [Cl III] & & & & 0.380 & 0.478 & 0.380 & 1.22 \\
\hline 553.8 & [Cl III] & & & & 0.535 & 0.603 & 0.535 & 1.72 \\
\hline 575.4 & [N II] & & & & 0.242 & 0.905 & 0.242 & 0.81 \\
\hline 587.6 & $\mathrm{He} \mathrm{I}$ & 23.80 & 15.6 & 15.1 & 20.15 & 14.10 & 18.66 & 60.9 \\
\hline 610.2 & [K IV] & & & & 0.042 & 0.016 & 0.042 & 0.14 \\
\hline 631.2 & [S II] & & & 0.74 & 0.912 & 1.309 & 0.826 & 2.74 \\
\hline 654.8 & {$[\mathrm{~N} \mathrm{II}]$} & 10.40 & 5.7 & & 4.884 & 21.97 & 6.99 & 23.3 \\
\hline 658.3 & {$[\mathrm{~N}$ II $]$} & 17.30 & 16.8 & 16.2 & 17.27 & 59.29 & 16.89 & 56.4 \\
\hline 671.6 & [S II] & & & 0.85 & 0.652 & 3.021 & 0.751 & 2.52 \\
\hline 673.1 & [S II] & & & 1.40 & 1.502 & 5.722 & 1.451 & 4.86 \\
\hline 713.6 & [Ar III] & 22.2 & & 14.0 & 21.18 & 17.45 & 19.13 & 64.8 \\
\hline 717.1 & [Ar IV] & & & & 0.026 & 0.025 & 0.026 & 0.09 \\
\hline 753.0 & [Cl IV] & & & & 0.144 & 0.057 & 0.144 & 0.49 \\
\hline 775.1 & [Ar III] & & & 3.0 & 4.674 & 4.589 & 3.837 & 16.10 \\
\hline 804.6 & [Cl IV] & & & & 0.300 & 0.110 & 0.300 & 1.05 \\
\hline 857.9 & {$[\mathrm{Cl} \mathrm{II}]$} & & & & 0.048 & 0.127 & 0.048 & 0.17 \\
\hline 953.1 & [S III] & & & & 19.10 & 46.46 & 19.10 & 68.4 \\
\hline
\end{tabular}

$\dagger$ References: MP: Manchado \& Pottasch (1989), M: from Middlemass et al. (1989), AC: Aller \& Czyzak (1979), H-east and H-north: Hyung et al. (2000).

${ }^{b}$ Blended with the line at $372.9 \mathrm{~nm}$.

not used when deriving the nebular ionic abundances. The low resolution spectrum is better calibrated and therefore these intensities are used.

There are several optical line studies in the literature. We selected those by Aller \& Czyzak (1979), Manchado \& Pottasch (1989), Middlemass et al. (1989) and Hyung et al. (2000). The first studied the central region, the second and third also the halo. As mentioned, Hyung et al. (2000) studied the East and North region of the nebula but only those of the eastern region were included. The objective of this paper is to study the chemical composition of the whole nebula. The lines in the east region resemble those of the central part and an average of those was used. Those of the north differ sometimes by more than $30 \%$, which probably indicates that inhomogeneities are present in the nebula. Nevertheless Hyung et al. (2000) found that within the errors the abundances resemble one another in both regions. How the difference between the northern and eastern (which is adopted) regions could affect the final abundances derived in this paper are discussed in Sect. 7.3. All lines are listed in Table 7. Average fluxes (except the north region) were used to compute the ionic abundances, listed in the last column. Note that the fluxes given in the articles are already corrected for extinction except those of Manchado \& Pottasch (1989) which we have corrected using $C_{\mathrm{H}_{\beta}}=0.2$ for comparison. Middlemass et al. (1989) used $C_{\mathrm{H}_{\beta}}=0.22$ and Aller \& Czyzak (1979) $C_{\mathrm{H}_{\beta}}=0.2$. Only Hyung et al. (2000) used a 
Table 8. Electron density in $\mathrm{cm}^{-3}$. For BD +303639 a $T_{\mathrm{e}}=8500 \mathrm{~K}$ has been used, and for NGC $6543 T_{\mathrm{e}}=8200 \mathrm{~K}$.

\begin{tabular}{lccccccc}
\hline \hline Ion & IP & \multicolumn{2}{c}{ Lines } & \multicolumn{2}{c}{ BD+303639 } & & \multicolumn{2}{c}{ NGC 6543 } \\
& $(\mathrm{eV})$ & used $(\mathrm{nm})$ & Ratio & $N_{\mathrm{e}}$ & & Ratio & $N_{\mathrm{e}}$ \\
\hline S III & 34.8 & $18700 / 33500$ & 5.0 & 10200 & & 2.6 & 3400 \\
O II & 35.1 & $372.6 / 372.9$ & 2.5 & 11300 & & 2.4 & $9300^{\dagger}$ \\
Cl III & 39.6 & $553.8 / 551.8$ & 3.1 & 48000 & & 1.4 & 6600 \\
\hline
\end{tabular}

$\dagger$ Uncertain density.

higher value of 0.3 . Nevertheless they mentioned that this is somewhat higher than previous determinations $(0.2)$ but they used 0.3 to compensate for the calibration, and therefore is a valid complement with the others. Since this average is corrected using $C_{\mathrm{H}_{\beta}}=0.2$ and we have found $C_{\mathrm{H}_{\beta}}=0.1$, in the last column the average fluxes using $C_{\mathrm{H}_{\beta}}=0.1$ have been listed and used to derive the abundances.

\section{Physical conditions}

Using these data, the nebular average physical parameters (electron temperature and density) have been derived.

\subsection{Electron density}

Three ions have been used to derive the electron density $\left(N_{\mathrm{e}}\right)$ for both nebulae and are shown in Table 8. An electron temperature of $8500 \mathrm{~K}$ (see Sect. 6.2) was used for BD+30 3639 and $T_{\mathrm{e}}=8200$ for NGC 6543 .

For BD+303639 the S III and O II ions give density values of 10200 and $11300 \mathrm{~cm}^{-3}$ respectively. This is in agreement with the value of Aller \& Hyung (1995) $\left(10000 \mathrm{~cm}^{-3}\right)$ and somewhat higher than the one found by Pottasch (1984). Keenan et al. (1999) calculated emission-line-ratio diagrams in [O II] using R-matrix calculations of electron impact excitation rates and obtain a somewhat lower density of $N_{\mathrm{e}}=$ $8000 \mathrm{~cm}^{-3}$. The line ratio for $\mathrm{Cl}$ III gives a very high value. This ion must be formed in a denser region and could indicate that clumps are present in the nebula. In order to determine the abundances an $N_{\mathrm{e}}\left(11000 \mathrm{~cm}^{-3}\right)$ average of these given by the $\mathrm{S}$ III and $\mathrm{O}$ II ions has been used, except for $\mathrm{Cl}$ III where $48000 \mathrm{~cm}^{-3}$ was used.

In the case of NGC 6543 the $N_{\mathrm{e}}$ found ranges from 3400 to $9300 \mathrm{~cm}^{-3}$, although the latter value has a high uncertainty. These three ions have the same $I P$ and the variation in $N_{\mathrm{e}}$ could be due to the presence of clump structures in the nebula. Images of the nebula indeed reveal some structure. For the determination of the abundances an average density of $5000 \mathrm{~cm}^{-3}$ from the S III and Cl III has been used which agrees with previous determinations (Hyung et al. 2000). The O II was avoided due to its larger uncertainty.

\subsection{Electron temperature}

To compute the electron temperature $\left(T_{\mathrm{e}}\right)$ lines ratios are needed originating from energy levels differing by several electron volts. For BD+303639 an electron density of $11000 \mathrm{~cm}^{-3}$ was assumed to derive the temperature and for
Table 9. Electron temperature in K. For BD+303639 a $N_{\mathrm{e}}=$ $11000 \mathrm{~cm}^{-3}$ has been used, and for NGC $6543 N_{\mathrm{e}}=5000 \mathrm{~cm}^{-3}$.

\begin{tabular}{ccccccccc}
\hline \hline Ion & IP & Lines & \multicolumn{2}{c}{ BD+303639 } & & \multicolumn{2}{c}{ NGC 6543 } \\
\cline { 7 - 8 } & $(\mathrm{eV})$ & used (nm) & Ratio & $T_{\mathrm{e}}$ & & Ratio & $T_{\mathrm{e}}$ \\
\hline N II & 14.5 & $658.3 / 575.4$ & 77.8 & 8400 & & 69.4 & 9500 \\
N II & 14.5 & $654.8 / 575.4$ & & & & 28.8 & 8800 \\
S III & 23.3 & $18700 / 631.2$ & 20.5 & $8600^{\dagger}$ & & 48.4 & 7800 \\
O III & 35.1 & $500.7 / 436.3$ & & & & 387 & 7800 \\
Ne III & 40.9 & $15500 / 386.9$ & & & & 4.0 & 8000 \\
Ar III & 27.6 & $8900 / 519.2$ & & & & 531 & $7500^{\dagger}$ \\
\hline
\end{tabular}

${ }^{\dagger}$ Large error.

NGC $6543 N_{\mathrm{e}}=5000 \mathrm{~cm}^{-3}$ (see Sect. 6.1). These values are listed in Table 9.

S III and N II have been used to derive $T_{\mathrm{e}}$ for BD+30 3639 . Both values agree and average out to $8500 \mathrm{~K}$. This is in good agreement with previous determinations by Aller \& Hyung (1995), Pottasch et al. (1986) and Keenan et al. (1999) who give an $T_{\mathrm{e}}$ of 8800,8000 and $8250 \mathrm{~K}$ respectively. For the purpose of this work an $T_{\mathrm{e}}=8500 \mathrm{~K}$ is assumed throughout the paper.

For NGC 6543 six ions were used to derive $T_{\mathrm{e}}$. N II (which has a lower $I P$ than the other ions) gives a higher temperature (around $9000 \mathrm{~K}$ ) than the rest of the ions which average out to $7800 \mathrm{~K}$. The indication of the N II needs to be confirmed. For the rest of the paper an average $T_{\mathrm{e}}=8200$ of all ions is assumed for NGC 6543.

\section{Abundances}

In Table 10 ionic and elemental abundances for BD+30 3639 and NGC 6543 are given. For most ions no abundance estimates for unseen stages of ionization are needed, thus the Ionization Correction Factor $(I C F)$ is 1. In Table 11, these abundances are compared with those found in the Sun, O-B stars, and in previous studies.

The helium abundances have been determined comparing the measured optical lines of $\mathrm{He}$ II at $468.6 \mathrm{~nm}$ and $\mathrm{He}$ I at 447.1 and $587.6 \mathrm{~nm}$, with theoretical predictions of Benjamin et al. (1999). The comparison with theory has been done for $N_{\mathrm{e}}=10000 \mathrm{~cm}^{-3}$ and an interpolation temperature of $8500 \mathrm{~K}$ for BD+30 3639 and $8200 \mathrm{~K}$ for NGC 6543 .

Taking into account the flux error the error in the abundances amounts to 10-25\% for all elements except helium. Other errors are difficult to quantify, such as those caused by atomic parameters (especially collisional strengths). Care was take when selecting them (see Bernard Salas et al. 2002). The use of ionization correction factors also introduces some uncertain error which increases with the $I C F$. In this study the $I C F$ were usually unnecessary but when needed were very small and therefore do not contribute much to the error. A quantitative idea of the error can be inferred in several ways. The present abundances are in most cases within $30 \%$ of other studies (except that of the carbon). The ionic abundances were derived in many cases using more than one line and these are consistent with each other to within 30\%. Argon and sulfur are elements not supposed to change in the course of evolution of 
Table 10. Ionic concentrations and chemical abundances.

\begin{tabular}{|c|c|c|c|c|c|c|c|c|c|}
\hline \multirow[t]{2}{*}{ Ion } & \multirow{2}{*}{$\begin{array}{c}\lambda \\
(\mathrm{nm}) \\
\end{array}$} & \multicolumn{4}{|c|}{$\mathrm{BD}+303639$} & \multicolumn{4}{|c|}{ NGC 6543 } \\
\hline & & $N_{\text {ion }} / N_{\mathrm{p}}$ & $\sum N_{\text {ion }} / N_{\mathrm{p}}$ & $I C F^{\natural}$ & $N_{\text {elem. }} / N_{\mathrm{p}}$ & $N_{\text {ion }} / N_{\mathrm{p}}$ & $\sum N_{\text {ion }} / N_{\mathrm{p}}$ & $I C F^{\natural}$ & $N_{\text {elem. }} / N_{\mathrm{p}}$ \\
\hline $\mathrm{He}^{0}$ & $447.2,587.5$ & $0.061^{\star}$ & & & & $0.118^{\star}$ & & & \\
\hline $\mathrm{He}^{+}$ & 468.6 & & & & & $4.0(-5)^{\star}$ & 0.118 & 1 & 0.118 \\
\hline $\mathrm{C}^{+}$ & 232.5 & $6.49(-4)$ & & & & $2.34(-5)$ & & & \\
\hline $\mathrm{C}^{++}$ & 190.9 & $8.42(-5)$ & $7.33(-4)$ & 1 & $7.3(-4)$ & $1.89(-4)$ & $2.12(-4)$ & 1.2 & $2.5(-4)$ \\
\hline $\mathrm{N}^{o}$ & $519.7,520.0$ & $2.16(-6)$ & & & & & & & \\
\hline $\mathrm{N}^{+}$ & $575.5,658.4$ & $1.07(-4)$ & & & & $8.70(-6)$ & & & \\
\hline $\mathrm{N}^{++}$ & $57300,174.5$ & $<1.70(-5)$ & & & & $2.13(-4)$ & & & \\
\hline $\mathrm{N}^{3+}$ & 148.5 & & $1.09(-4)$ & 1 & $1.1(-4)$ & $1.13(-5)$ & $2.33(-4)$ & 1 & $2.3(-4)$ \\
\hline $\mathrm{O}^{o}$ & $630.0,636.3$ & $5.32(-6)$ & & & & & & & \\
\hline $\mathrm{O}^{+}$ & 372.7 & $4.47(-4)$ & & & & $1.99(-5)$ & & & \\
\hline $\mathrm{O}^{++}$ & $495.9,500.7$ & $4.13(-6)$ & & & & $5.26(-4)$ & & & \\
\hline $\mathrm{O}^{3+}$ & 25890 & & $4.56(-4)$ & 1 & $4.6(-4)$ & $<6.03(-8)$ & $5.46(-4)$ & 1 & $5.5(-4)$ \\
\hline $\mathrm{Ne}^{+}$ & 12800 & $1.73(-4)$ & & & & $1.21(-5)$ & & & \\
\hline $\mathrm{Ne}^{++}$ & 15500,36010 & $<6.80(-7)$ & & & & $1.66(-4)$ & & & \\
\hline $\mathrm{Ne}^{3+}$ & 241.9 & & $1.73(-4)$ & 1.13 & $1.90(-4)$ & $1.31(-5)$ & $1.91(-4)$ & 1 & $1.9(-4)$ \\
\hline $\mathrm{S}^{+}$ & $671.6,673.0$ & $1.32(-6)$ & & & & $1.94(-7)$ & & & \\
\hline $\mathrm{S}^{++}$ & 18700,33400 & $5.07(-6)$ & & & & $7.24(-6)$ & & & \\
\hline $\mathrm{S}^{3+}$ & 10500 & $<2.60(-8)$ & $6.39(-6)$ & 1 & $6.4(-6)$ & $5.37(-6)$ & $1.28(-5)$ & 1 & $1.3(-5)$ \\
\hline $\mathrm{Cl}^{+}$ & $857.8,912.6$ & $4.11(-8)$ & & & & $4.58(-9)$ & & & \\
\hline $\mathrm{Cl}^{++}$ & $551.9,553.9$ & $9.55(-8)$ & & & & $1.48(-7)$ & & & \\
\hline $\mathrm{Cl}^{3+}$ & $11760,753.0$ & $<5.10(-8)$ & $1.37(-7)$ & 1 & $1.4(-7)$ & $5.03(-8)$ & $2.03(-7)$ & 1 & $2.0(-7)$ \\
\hline $\mathrm{Ar}^{+}$ & 6980 & $4.69(-6)$ & & & & $1.33(-7)$ & & & \\
\hline $\mathrm{Ar}^{++}$ & 8990 & $2.76(-7)$ & & & & $3.34(-6)$ & & & \\
\hline $\mathrm{Ar}^{3+}$ & $471.1,474.0$ & & $4.97(-6)$ & 1.04 & $5.2(-6)$ & $7.02(-7)$ & $4.19(-6)$ & 1 & $4.2(-6)$ \\
\hline
\end{tabular}

${ }^{\natural}$ ICF: Ionization Correction Factor.

^ Using the predictions for the helium emission lines given by Benjamin et al. (1999).

Table 11. Comparison of abundances in BD+303639 and NGC 6543.

\begin{tabular}{|c|c|c|c|c|c|c|c|}
\hline \multirow[t]{2}{*}{ Element } & \multicolumn{2}{|c|}{ BD+303639 } & \multicolumn{3}{|c|}{ NGC 6543 } & \multirow[t]{2}{*}{$\operatorname{Sun}^{1}$} & \multirow[t]{2}{*}{$\overline{\mathrm{O}, \mathrm{B} \text { Stars }}$} \\
\hline & Present abun. & $\mathrm{AH}^{3}$ & Present abun. & $\mathrm{Mid}^{4}$ & $\mathrm{HA}^{5}$ & & \\
\hline Helium & & 0.021 & 0.118 & 0.11 & 0.130 & 0.098 & \\
\hline Carbon $(-4)$ & 7.3 & 3.60 & 2.5 & 7.6 & 2.5 & 3.55 & 1.74 \\
\hline Nitrogen $(-4)$ & 1.1 & 1.16 & 2.3 & 0.84 & 1.2 & 0.93 & 0.65 \\
\hline Oxygen $(-4)$ & 4.6 & 3.78 & 5.5 & 6.4 & 4.7 & 4.90 & 4.17 \\
\hline Neon $(-4)$ & 1.9 & $>0.018$ & 1.9 & 1.6 & 1.0 & 1.20 & 1.23 \\
\hline Sulfur $(-5)$ & 0.64 & 0.71 & 1.3 & & 0.8 & 1.86 & 1.23 \\
\hline Chlorine $(-7)$ & 1.4 & 1.44 & 2.0 & & 1.6 & 1.86 & 1.86 \\
\hline $\operatorname{Argon}(-6)$ & 5.2 & $>0.2$ & 4.2 & & 3.0 & 3.63 & \\
\hline
\end{tabular}

${ }^{1}$ Solar abundance from Grevesse \& Noels (1993) and Anders \& Grevesse (1989), except the oxygen abundance that has been taken from Allende Prieto et al. (2001).

${ }^{2}$ O, B star abundances are the average of Gies \& Lambert (1992) and Killian-Montenbruck et al. (1994).

${ }^{3}$ From Aller \& Hyung (1995).

${ }^{4}$ From Middlemass et al. (1989).

${ }^{5}$ From Hyung et al. (2000).

intermediate mass stars. A comparison of these elements with other $\mathrm{PNe}$ in the literature agree within $30 \%$. We therefore think that $30 \%$ is a good estimate for the error on the abundances of all elements with the exception of the nitrogen in NGC 6543, which is around 50\%. The main nitrogen contribution in NGC 6543 comes from $\mathrm{N}^{++}$. This ionic abundance has been derived from the $57.3 \mu \mathrm{m}$ and $175.0 \mathrm{~nm}$ lines and is $3.1(-4)^{1}$ and $1.2(-4)$ respectively. Unfortunately the infrared line is density dependent and the ultraviolet line is temperature dependent, making it difficult to assess which one gives the best value, and increasing therefore the error. In this work an averaged $\mathrm{N}^{++}$ionic abundance from the infrared and ultraviolet lines has been used, and a larger error assumed. The error on the helium abundance for NGC 6543 is around $7 \%$.

\footnotetext{
${ }^{1}$ In this section and Tables 10,11 and 12 the number in parenthesis means: $x(y)=x \times 10^{y}$.
} 
Table 12. Comparison of the abundance ratios of carbon, nitrogen and oxygen.

\begin{tabular}{ccccc}
\hline \hline & BD+303639 & NGC 6543 & Sun & O Stars \\
\hline $\mathrm{N} / \mathrm{O}$ & 0.24 & 0.42 & 0.19 & 0.15 \\
$\mathrm{C} / \mathrm{O}$ & 1.59 & 0.45 & 0.72 & 0.42 \\
$\frac{(\mathrm{C}+\mathrm{N}+\mathrm{O})}{\mathrm{H}}(-4)$ & 13.0 & 10.3 & 9.4 & 6.56 \\
\hline
\end{tabular}

\section{1. $B D+303639$}

The elemental abundances for seven elements have been derived. High stages of ionization are not seen, as expected. To investigate their possible contribution upper limits for the unseen $\mathrm{N}^{++}, \mathrm{Ne}^{++}, \mathrm{S}^{3+}$ and $\mathrm{Cl}^{3+}$ were derived. Even a contribution to the abundance equal to each upper limit in $\mathrm{Ne}^{++}, \mathrm{S}^{3+}$, $\mathrm{Cl}^{3+}$, is negligible. $\mathrm{Ar}^{3+}$ and $\mathrm{C}^{3+}$ have higher $I P$ than for instance $\mathrm{S}^{3+}$, therefore no contribution is expected. The upper limit to $\mathrm{N}^{++}$is at most $15 \%$ that of the total. The $\mathrm{O}^{++}$, which has similar $I P$, contributes less than $1 \%$. Therefore we have assumed that $\mathrm{N}^{++}$does not contribute to the abundance of nitrogen. Even considering a $15 \%$ of contribution will be within the errors that have been estimated to be $30 \%$. Corrections for low unseen stages of ionization should also be estimated. Neutral nitrogen and oxygen have been measured (very low abundance) and can help in deriving the neutral contribution of the other ions. The $\mathrm{N}^{0} / \mathrm{N}^{+}$and the $\mathrm{O}^{0} / \mathrm{O}^{+}$ratio is 0.023 and 0.01 respectively. The $I P$ of nitrogen is $14.5 \mathrm{eV}$ and that of oxygen $13.6 \mathrm{eV}$. Using this information and the $I P$ of the ions of interest the contribution can be inferred. These are the following: $\mathrm{C}^{0} / \mathrm{H}=6.0(-7) ; \mathrm{Ne}^{0} / \mathrm{H}=1.26(-5) ; \mathrm{S}^{0} / \mathrm{H}=<1.3(-8)$; $\mathrm{Ar}^{0} / \mathrm{H}=2.0(-7) ; \mathrm{Cl}^{0} / \mathrm{H}<5(-10)$. It can be seen that the contribution is very small in all of them and negligible in carbon, sulfur and chlorine. For neon and argon this contribution has been taken into account and is reflected in the ICF used in Table 10. The helium abundance hasn't been derived because some of the helium is probably neutral.

A comparison of these abundances with those by Aller \& Hyung (1995) is given in Table 11. The nitrogen, sulfur and chlorine abundances agree very well. Aller \& Hyung (1995) gave a lower limit for argon and neon because they could only measure one ionization stage ( $\mathrm{Ne}$ II and Ar III). In this work we have been able to set an upper limit to the Ne III, which turns out to be negligible. The Ar II has been measured and is the major contributor to the total abundance of argon. Using the infrared line at $6.9 \mu \mathrm{m}$, an abundance of [Ar II] $=4.7(-6)$ is found; Aller \& Hyung (1995) give an upper limit of 2.0(-7) for the argon abundance using optical data. The oxygen abundance is higher, although within the errors, and can probably be explained by the different extinction used.

The abundances have been compared to those of the Sun and O, B stars in Tables 11 and 12. The abundances of nitrogen and oxygen are very similar to solar. The carbon abundance is larger and the $\frac{(\mathrm{C}+\mathrm{N}+\mathrm{O})}{\mathrm{H}}$ ratio is also larger than solar due to the enhanced carbon. The $\mathrm{N} / \mathrm{O}$ and $\mathrm{C} / \mathrm{O}$ ratios are much higher than in the Sun and for O, B stars. Processes such as the second dredge-up and hot bottom burning, in which carbon is destroyed, can not have occurred. Since these processes need certain mass to occur this could set a limit on the progenitor mass to be lower than $3 M_{\odot}$.

\subsection{NGC 6543}

The elemental abundances for 8 elements have been calculated and are given in Table 10. The ICF turns out to be 1 for seven cases. For carbon the $I C F$ is 1.2 because some $\mathrm{C}^{3+}$ might be present; but, considering its $I P$, and the fact that the $\mathrm{O}^{3+}$ (with an $I P$ of $54 \mathrm{eV}$ ) is not seen, it is expected that contribution of that stage of ionization should be small.

These abundances are compared in Table 11 with those of Middlemass et al. (1989) and Hyung et al. (2000). The helium, neon and oxygen agree within the errors from those found by Middlemass et al. (1989). On the other hand they found more carbon and less nitrogen. Compared with those by Hyung et al. (2000) carbon, oxygen, sulfur and argon are similar but the present nitrogen is again higher. The difference in the nitrogen abundance is probably related to the large uncertainty in the ionic abundance of $\mathrm{N}^{++}$, which has been assumed to be the average of that given by the optical and ultraviolet lines. Using the infrared line or ultraviolet line to derive the $\mathrm{N}^{++}$abundance leads to a $\mathrm{N} / \mathrm{H}$ of $3.3(-4)$ or $1.4(-4)$ respectively. The latter value is therefore in agreement with previous determinations. As has been explained before the ultraviolet line is temperature dependent and therefore we have preferred to used an average abundance and assume a larger error for this element.

When compared to the sun or O, B stars it is seen that the abundances resemble more those of the sun. Nitrogen is somewhat larger and carbon is lower. This could mean that some carbon has been converted into nitrogen in the course of evolution. The ratios of the abundances in Table 12 show that $\frac{(\mathrm{C}+\mathrm{N}+\mathrm{O})}{\mathrm{H}}$ is solar and could suggest a one solar mass progenitor for this nebula, in which via the CNO cycle some carbon has been destroyed to produce nitrogen.

\subsection{Inhomogeneities in NGC 6543}

In order to derive the chemical abundances for NGC 6543 three data sets, infrared, ultraviolet and optical have been combined. The purpose of this paper is to derive the abundances for the whole nebula. The infrared and ultraviolet observations cover almost the entire nebula, this is not the case for the optical data. This has been scaled to the entire $\mathrm{H}_{\beta}$ flux and considered representative of the entire nebula. The optical fluxes of the central and east regions are very similar but differ sometimes from those of the north.

To evaluate the difference we derived the ionic abundances for the three different regions. The abundances from the central and east part of the nebula agree between each other and support the fact that they were combined to give the ionic abundances. Compared to the North region there are sometimes ions that show differences higher than $30 \%$. None of these ions (except the S III) are the principal stage of ionization, that is the major contribution to the element abundance doesn't come for that ion. For example, O II contributes only $7 \%$ to the total 
oxygen abundance, much smaller than the error in the value of the abundance itself. Thus the difference in fluxes of the different regions of such ions does not affect the final element abundance determination. The S III $(\lambda 631.2,953.1)$ exhibit an ionic abundance difference higher than $30 \%$ and this stage of ionization is the main contributor to the total one. Nevertheless, for this ion the ionic abundance have been derived using the infrared lines at 18.7 and $33.4 \mu \mathrm{m}$, thus avoiding this uncertainty.

\section{Conclusions}

Reliable infrared spectra have been observed for BD+30 3639 and NGC 6543. Newly reduced high and low resolution IUE data for NGC 6543 are also presented. This has been complemented with optical (and ultraviolet for BD+30 3639) lines from the literature in order to accurately determine the elemental abundances for the nebulae.

Also, the electron temperature and density of the nebulae have been determined. The average electron temperature and density in $\mathrm{BD}+303639$ are $T_{\mathrm{e}}=8800 \mathrm{~K}$ and $N_{\mathrm{e}}=11000 \mathrm{~cm}^{-3}$, which agree with previous studies. For NGC $6543 T_{\mathrm{e}}=8500 \mathrm{~K}$ and $N_{\mathrm{e}}=5000 \mathrm{~cm}^{-3}$ have been found.

The abundances for 6 elements in BD+303639 and 8 for NGC 6543 have been derived and are given in Table 10. Ionization corrections factors turned out to be very small, and in most cases are unity, indicating a high accuracy $(\sim 30 \%)$ in the abundance determinations. The abundances of $\mathrm{BD}+303639$ are similar to solar except for carbon, which is larger. This could indicate that the second dredge-up and hot bottom burning (processes in which carbon is destroyed) have not occurred. If this is true it could set a lower limit to the mass of the progenitor star (around $3 M_{\odot}$ ) above which these processes take place. The abundances of NGC 6543 also resemble those of the sun. The $\frac{(\mathrm{C}+\mathrm{N}+\mathrm{O})}{\mathrm{H}}$ ratio suggests that the progenitor star could be a one solar mass star where some carbon has been converted into nitrogen as indicated by the $\mathrm{C} / \mathrm{O}$ and $\mathrm{N} / \mathrm{O}$ ratios.

Acknowledgements. IA3 is a joint development of the SWS consortium. Contributing institutes are SRON, MPE, KUL and the ESA Astrophysics Division.

\section{References}

Acker, A., Ochsenbein, F., Stenholm, B., et al. 1992, Catalogue of Galactic Planetary Nebulae-Part II, Strasbourgh-ESO

Allende Prieto, C., Lambert, D. L., \& Asplund, M. 2001, ApJ, 556, L63

Aller, L. H., \& Czyzak, S. J. 1979, Ap\&SS, 62, 397

Aller, L. H., \& Hyung, S. 1995, MNRAS, 276, 1101

Anders, E., \& Grevesse, N. 1989, Geochem. Cosmo., 53, 197

Beintema, D. A., \& Pottasch, S. R. 1999, A\&A, 347, 942

Benjamin, R. A., Skillman, E. D., \& Smits, D. P. 1999, ApJ, 514, 307

Bernard Salas, J., Pottasch, S. R., Feibelman, W. A., \& Wesselius, P. R. 2002, A\&A, 387, 301

Bernard Salas, J., Pottasch, S. R., Beintema, D. A., \& Wesselius, P. R. 2001, A\&A, 367, 949

Castor, J. I., Lutz, J. H., \& Seaton, M. J. 1981, MNRAS, 194, 547

Clegg, P. E., Ade, P. A. R., Armand, C., et al. 1996, A\&A, 315, L38

de Graauw, T., Haser, L. N., Beintema, D. A., et al. 1996, A\&A, 315, L49

Fluks, M. A., Plez, B., Thé, P. S., et al. 1994, A\&AS, 105, 311

George, D., Kaftan-Kassim, M. A., \& Hartsuijker, A. P. 1974, ApJ, 35, 219

Gies, D. R., \& Lambert D. L. 1992, ApJ, 387, 673

Grevesse, N., \& Noels, A. 1993, in Origin and Evolution of the Elements, ed. N. Prantos, E. Vangioni-Flam, \& M. Cassé (Cambridge University Press), 15

Guerrero, M. A., Chu, Y. -H., Gruendl, R. A., Williams, R. M., \& Kaler, J. B. 2001, ApJ, 553, L55

Hummer, D. G., \& Storey, P. J., 1987, MNRAS, 224, 801

Hyung, S., Aller, L. H., Feibelman, W. A., Lee, W. B., \& de Koter, A. 2000, MNRAS, 318, 77

Kastner, J. H., Soker, N., Vrtilek, S. D., \& Dgani, R. 2000, ApJ, 545, L57

Keenan, F. P., Aller, L. H., Bell, K. L., et al. 1999, MNRAS, 304,27

Killian-Montenbruck J., Grehen T., \& Nissen P. E. 1994, ApJ, 291, 757

Manchado, A., \& Pottasch, S. R. 1989, A\&A, 222, 219

Middlemass, D., Clegg, E. S., \& Walsh, J. R. 1989, MNRAS, 239, 1

Persi, P., Cesarsky, D., Marenzi, A. R., et al. 1999, A\&A, 351, 201

Pottasch, S. R., 1984, Planetary Nebulae, D-Reidel, vol. 107

Pottasch, S. R., Preite-Marinez, A., Olnon, F. M., Mo, J. -E., \& Kingma, S. 1986, A\&A, 161, 363

Pwa, T. H., Pottasch, S. R., \& Mo, J. E. 1986, A\&A, 164, 184

Torres-Peimbert, S., \& Peña, M. 1981, Rev. Mex. Astron. Astrofis., 6, 301

Rudy, R. J., Rossano, G. S., Erwin, P., et al. 1991, ApJ, 380, 151 